\title{
Correction to: Factors influencing liberation from mechanical ventilation in coronavirus disease 2019: multicenter observational study in fifteen Italian ICUs
}

Lorenzo Gamberini ${ }^{1}$, Tommaso Tonetti ${ }^{2 *}$, Savino Spadaro ${ }^{3}$, Gianluca Zanii ${ }^{4}$, Carlo Alberto Mazzolii , Chiara Capozzi ${ }^{5}$, Emanuela Giampalma ${ }^{6}$, Maria Letizia Bacchi Reggiani ${ }^{7}$, Elisabetta Bertellini ${ }^{8}$, Andrea Castelli ${ }^{5}$, Irene Cavalli Davide Colombo ${ }^{9,10}$, Federico Crimaldi ${ }^{11}$, Federica Damiani ${ }^{12}$, Alberto Fogagnolo ${ }^{3}$, Maurizio Fusari ${ }^{4}$, Emiliano Gamberini ${ }^{13}$, Giovanni Gordini ${ }^{1}$, Cristiana Laici ${ }^{14}$, Maria Concetta Lanza ${ }^{15}$, Mirco Leo ${ }^{16}$, Andrea Marudi ${ }^{8}$, Giuseppe Nardi ${ }^{17}$, Irene Ottaviani ${ }^{3}$, Raffaella Papa ${ }^{18}$, Antonella Potalivo ${ }^{17}$, Emanuele Russo ${ }^{13}$, Stefania Taddei ${ }^{19}$, Carlo Alberto Volta ${ }^{3}$, V. Marco Ranieri ${ }^{2}$ and the ICU-RER COVID-19 Collaboration

\section{Correction to: J Intensive Care (2020) 8:80} https://doi.org/10.1186/s40560-020-00499-4

Following publication of the original article [1], the authors identified an error in the collaborator author's name of Elena Mosconi.

The incorrect author name is: Giulia Mosconi

The correct author name is: Elena Mosconi

The original article has been updated.

\section{Author details}

'Department of Anaesthesia, Intensive Care and Prehospital Emergency, Ospedale Maggiore Carlo Alberto Pizzardi, Bologna, Italy. ${ }^{2}$ Alma Mater Studiorum, Dipartimento di Scienze Mediche e Chirurgiche, Anesthesia and Intensive Care Medicine, Policlinico di Sant'Orsola, Università di Bologna, Bologna, Italy. ${ }^{3}$ Department of Morphology, Surgery and Experimental Medicine, Section of Anaesthesia and Intensive Care University of Ferrara, Azienda Ospedaliero-Universitaria S. Anna, Via Aldo Moro, 8, 44121 Ferrara, Cona, Italy. ${ }^{4}$ Department of Anesthesia and Intensive Care, Santa Maria delle Croci Hospital, Ravenna, Italy. ${ }^{5}$ Cardio-Anesthesiology Unit,

Cardio-Thoracic-Vascular Department, S.Orsola Hospital, University of Bologna, Bologna, Italy. ${ }^{6}$ Radiology Department, M.Bufalini Hospital, Cesena, Italy. ${ }^{7}$ Alma Mater University, Department of Clinical, Integrated and

\section{The original article can be found online at https://doi.org/10.1186/s40560} 020-00499-4

*Correspondence: tommaso.tonetti@unibo.it

${ }^{2}$ Alma Mater Studiorum, Dipartimento di Scienze Mediche e Chirurgiche,

Anesthesia and Intensive Care Medicine, Policlinico di Sant'Orsola, Università di Bologna, Bologna, Italy

Full list of author information is available at the end of the article
Experimental Medicine (DIMES), Statistical Service, S. Orsola-Malpighi Hospital Bologna, Bologna, Italy. ${ }^{8}$ Department of Anaesthesiology, University Hospital of Modena, Via del Pozzo 71, 41100 Modena, Italy. ${ }^{9}$ Anaesthesia and Intensive Care Department, SS. Trinità Hospital, ASL, Novara, Italy. ${ }^{10}$ Translational Medicine Department, Eastern Piedmont University, Novara, Italy. ${ }^{11}$ Anaesthesia and Intensive Care Residency Program - Translational Medicine Department, Eastern Piedmont University, Novara, Italy.

${ }^{12}$ Department of Anaesthesia, Intensive Care and Pain Therapy - Imola Hospital, Imola, Italy. ${ }^{13}$ Anaesthesia and Intensive Care Unit, M. Bufalini Hospital, Cesena, Italy. ${ }^{14}$ Division of Anesthesiology, Hospital S. Orsola Malpighi, Alma Mater Studiorum University of Bologna, Bologna, Italy. ${ }^{15}$ Department of Anesthesia and Intensive Care, G.B. Morgagni-Pierantoni Hospital, Forli, Italy. ${ }^{16}$ Department of Anaesthesia and Intensive Care, Azienda Ospedaliera SS. Antonio e Biagio e Cesare Arrigo, Alessandria, Italy.

${ }^{17}$ Department of Anaesthesia and Intensive Care, Infermi Hospital, Rimini, Italy. ${ }^{18}$ Anaesthesia and Intensive Care Unit, Santa Maria Annunziata Hospital, Firenze, Italy. ${ }^{19}$ Anaesthesia and Intensive Care Unit, Bentivoglio Hospital, Bentivoglio, Italy.

Published online: 17 December 2020

\section{Reference}

1. Gamberini L, et al. Factors influencing liberation from mechanical ventilation in coronavirus disease 2019: multicenter observational study in fifteen Italian ICUs. J Intensive Care. 2020;8:80.

(c) The Author(s). 2020 Open Access This article is licensed under a Creative Commons Attribution 4.0 International License, which permits use, sharing, adaptation, distribution and reproduction in any medium or format, as long as you give appropriate credit to the original author(s) and the source, provide a link to the Creative Commons licence, and indicate if changes were made. The images or other third party material in this article are included in the article's Creative Commons. licence, unless indicated otherwise in a credit line to the material. If material is not included in the article's Creative Commons licence and your intended use is not permitted by statutory regulation or exceeds the permitted use, you will need to obtain permission directly from the copyright holder. To view a copy of this licence, visit http://creativecommons.org/licenses/by/4.0/ The Creative Commons Public Domain Dedication waiver (http://creativecommons.org/publicdomain/zero/1.0/) applies to the data made available in this article, unless otherwise stated in a credit line to the data. 